\title{
Variation between centres in access to renal transplantation in UK: longitudinal cohort study
}

\author{
R Ravanan, consultant nephrologist, ${ }^{1}$ U Udayaraj, consultant nephrologist , , D Ansell, director, ${ }^{2}$ D Collett, \\ director of statistics and audit, ${ }^{3} \mathrm{R}$ Johnson, principal statistician, ${ }^{3}$ J O'Neill, statistician, ${ }^{3}$ C R V Tomson, chair \\ and consultant nephrologist, ${ }^{2}$ C R K Dudley, consultant nephrologist ${ }^{1}$
}

\section{'Richard Bright Renal Unit, \\ Southmead Hospital, Bristol BS10 \\ $5 \mathrm{NB}$ \\ ${ }^{2}$ UK Renal Registry, Southmead Hospital, Bristol BS10 5NB \\ ${ }^{3}$ Organ Donation and \\ Transplantation Directorate, Bristol BS34 8RR \\ Correspondence to: $\mathrm{R}$ Ravanan Rommel.Ravanan@nbt.nhs.uk \\ Cite this as: $B M J$ J 2010;341:C3451 doi:10.1136/bmj.c3451}

\section{ABSTRACT}

Objective To assess whether equity exists in access to renal transplantation in the UK after adjustment for case mix in incident patients with end stage renal disease. Design Longitudinal cohort study.

Setting UK Renal Registry and UK Transplant Registry. Participants All incident renal replacement treatment patients $(n=16202)$ from 65 renal centres submitting data to the UK Renal Registry between 1 January 2003 and 31 December 2005, followed until 31 December 2008 (or until transplantation or death, whichever was earliest). Outcome measures Proportion of incident dialysis patients at each renal centre who were registered on the national transplant list; time taken to achieve registration; and proportion of patients subsequently transplanted.

Results We found that recipients' age, ethnicity, and primary renal diagnosis were associated with the likelihood of accessing the waiting list or receiving a transplant. After adjustment for case mix, significant inter-centre variability existed in access to the transplant list (change in $-2 \log L=89.9, \mathrm{df}=1, \mathrm{P}<0.001$ ), in the time taken to register patients on the waiting list (change in $-2 \log L=247.4, d f=64, P<0.001)$, in receipt of a renal transplant from a donor after brain stem death (change in $-2 \log L=15.1, d f=1, P=0.001$ ), and in receipt of a renal transplant from a living donor or a donor after cardiac death (change in -2LogL=46.1, df=1, P<0.001).

Conclusions Significant variation in access to renal transplantation exists between centres within the UK that cannot be explained by differences in case mix.

\section{INTRODUCTION}

For suitable patients with end stage renal disease, renal transplantation is accepted as the optimal modality of renal replacement treatment, conferring both better quality of life and better life expectancy than dialysis. However, deciding which patients are "suitable" for renal transplantation requires an individualised assessment of the risks of transplantation as well as the likely benefit. A renal transplant may be obtained from a donor after brain stem death, a donor after cardiac death, or a living kidney donor. To obtain a renal transplant from a donor after brain stem death, patients with end stage renal disease need to be registered on the national transplant list. Once registered, individual patients are offered a transplant according to a national allocation algorithm (www.uktransplant.org.uk/ukt/ about_transplants/organ_allocation/kidney_(renal)/ kidney_(renal).jsp). Transplantation from a living kidney donor requires that potential donors are identified and medically evaluated. Finally, transplantation from a donor after cardiac death in the United Kingdom is predominantly locally resourced, and allocation of organs is controlled by local centres' policies. The probability of receiving a transplant from a donor after brain stem death once a patient is on the waiting list is thus predominantly under the influence of national organ allocation algorithms, whereas the probability of receiving a transplant from a donor after cardiac death or a living kidney donor is predominantly influenced by individual centres' policies and patterns of practice.

Many patient specific factors including age, sex, ethnicity, and comorbidity have been reported to influence access to kidney transplantation in countries outside the UK. ${ }^{1-10}$ In the United States, insurance status has also been reported as an independent predictive variable determining access to the renal transplant list. ${ }^{11}$ Although general guidelines for the assessment of patients' suitability for transplantation are available, individual clinicians' and centres' practices may vary in the interpretation of these guidelines. Studies examining centre specific differences in access to renal transplantation are limited. ${ }^{12}$ Within the UK, a significant effect of centre on the likelihood of being put on the waiting list for renal transplantation for incident patients in Scotland and among prevalent dialysis patients in England and Wales has been described. ${ }^{13} 14$ Finally, whether differences exist between centres that influence the likelihood of subsequent kidney transplantation in patients registered on the national transplant list is not known.

Time on dialysis is recognised as an important prognostic factor that adversely influences the survival of grafts and patients after transplantation. ${ }^{15}$ The time taken to register a suitable patient on the transplant waiting list is mainly influenced by a centre's practice 
Table 1| Results of logistic regression modelling for probability of activation on waiting list within two years of start of renal replacement treatment

\begin{tabular}{|c|c|c|c|}
\hline Baseline factor & No & Odds ratio $(95 \% \mathrm{Cl})$ & $P$ value \\
\hline \multicolumn{4}{|l|}{ Age (years): } \\
\hline $18-29$ & 766 & 1 (referent) & NA \\
\hline $30-39$ & 1215 & 0.75 (0.61 to 0.93$)$ & 0.007 \\
\hline $40-49$ & 1841 & $0.50(0.41$ to 0.61$)$ & $<0.001$ \\
\hline $50-59$ & 2448 & 0.25 (0.20 to 0.30$)$ & $<0.001$ \\
\hline $60-64$ & 1593 & 0.11 (0.09 to 0.13$)$ & $<0.001$ \\
\hline \multicolumn{4}{|l|}{ Ethnicity: } \\
\hline White & 4908 & 1 (referent) & NA \\
\hline Non-white & 1284 & 0.85 (0.74 to 0.97$)$ & 0.02 \\
\hline Missing & 1671 & 0.74 (0.66 to 0.84$)$ & $<0.001$ \\
\hline \multicolumn{4}{|l|}{ Sex: } \\
\hline Male & 4748 & 1 (referent) & NA \\
\hline Female & 3115 & 0.98 (0.89 to 1.09$)$ & 0.75 \\
\hline \multicolumn{4}{|c|}{ Primary renal diagnosis: } \\
\hline Non-diabetes & 5765 & 1 (referent) & NA \\
\hline Diabetes & 1757 & $0.40(0.36$ to 0.45$)$ & $<0.001$ \\
\hline Missing & 341 & 0.55 (0.43 to 0.69$)$ & $<0.001$ \\
\hline
\end{tabular}

$\mathrm{NA}=$ not applicable. proportion transplanted, we followed all patients from the incident cohort who were activated on the waiting list before 31 December $2006(n=4061)$ until 31 December 2008, to estimate the proportion transplanted with a kidney alone or kidney plus pancreas within two years of inclusion on the waiting list.

We excluded patients aged 65 years or above because only a small proportion $(6 \%)$ of the 8210 patients aged 65 or older who started dialysis in 20035 were ever subsequently activated on the transplant list. We considered only centres contributing data to the UK Renal Registry for inclusion because we had no reliable mechanism for identifying or recording the patient level data we needed for patients starting renal replacement treatment in units $(\mathrm{n}=7)$ not linked to the registry. Previous analysis at the UK Renal Registry (David Ansell, personal communication, 2002) had found that several centres had a practice of activating patients on the transplant list followed by immediate suspension before more permanent activation at a later date after more formal medical assessment of the patient's fitness. We therefore excluded patients who were suspended for more than 30 days within 90 days of first activation to prevent this activation practice influencing the results.

Information on start date of renal replacement treatment and relevant patient level data including age (grouped as 18-29, 30-39, 40-49, 50-59, and $\geq 60$ ), sex, ethnicity (white, non-white, and missing), and primary renal diagnosis (diabetes, non-diabetes, and missing) came from the UK Renal Registry; the date of activation on the waiting list, date of transplantation, or both came from the UK Transplant Registry held by the Organ Donation and Transplantation Directorate of NHS Blood and Transplant. The UK Renal Registry is part of the Renal Association, a registered charity established for the development of care of patients with renal disease. The registry acts as a source of comparative data for audit, benchmarking, planning, policy, and research. The UK Renal Registry is funded by

ing list, and whether equity exists in the receipt of renal transplant once the patient is on the transplant list (that is, the conversion efficiency from being on the waiting list to receiving a transplant). We analysed patient specific and independent variables that influenced access to the waiting list or transplantation.

\section{METHODS}

\section{Study population}

We considered for inclusion all patients starting renal replacement treatment $(\mathrm{n}=16202)$ between 1 January 2003 and 31 December 2005 in renal centres returning data to the UKRenal Registry $(\mathrm{n}=65)$. For the analysis of the proportion included on the waiting list, we excluded patients aged 65 years or above $(\mathrm{n}=8210)$, inappropriate activation with early suspension as described below $(\mathrm{n}=106)$, and patients listed for multi-organ transplants other than pancreas $(n=23)$, resulting in a final cohort of 7863 patients. We followed all patients to 31 December 2007 or until they were put on the waiting list for kidney transplant alone, kidney plus pancreas transplant, or death, whichever was earliest. For the analysis of the

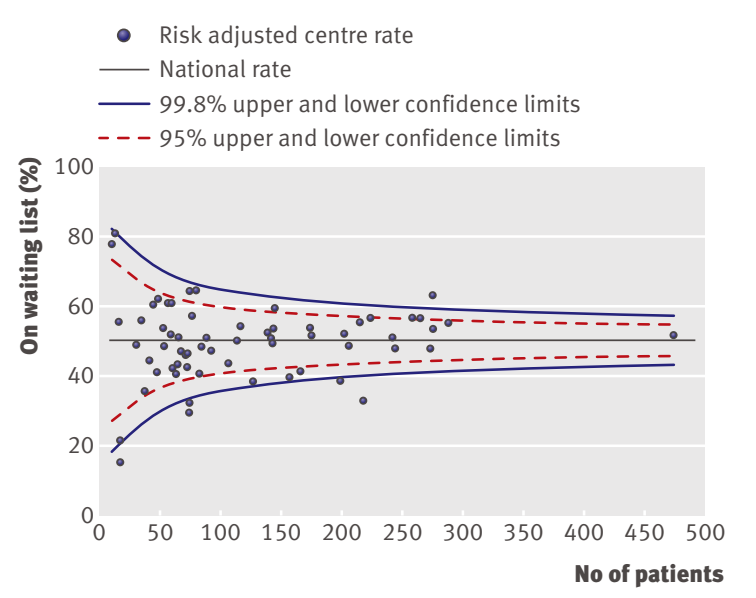

Fig 1| Percentage registered for transplantation within two years of starting renal replacement treatment. Risk adjusted for effect of recipient's age, sex, ethnicity, and primary renal diagnosis 
Table 2 |Results of logistic regression modelling for probability of receiving transplant from donor after brain stem death within two years of registration on waiting list

\begin{tabular}{|c|c|c|c|}
\hline Baseline factor & No & Odds ratio $(95 \% \mathrm{Cl})$ & $P$ value \\
\hline \multicolumn{4}{|l|}{ Age (years): } \\
\hline $18-29$ & 600 & 1 (referent) & NA \\
\hline $30-39$ & 853 & $1.13(0.89$ to 1.44$)$ & 0.32 \\
\hline $40-49$ & 1120 & 0.99 (0.78 to 1.25$)$ & 0.92 \\
\hline $50-59$ & 1062 & $0.69(0.54$ to 0.87$)$ & 0.002 \\
\hline $60-64$ & 426 & $0.44(0.31$ to 0.61$)$ & $<0.001$ \\
\hline \multicolumn{4}{|l|}{ Ethnicity: } \\
\hline White & 2645 & 1 (referent) & NA \\
\hline Non-white & 675 & 0.47 (0.37 to 0.59$)$ & $<0.001$ \\
\hline Missing & 741 & 0.91 (0.75 to 1.10$)$ & 0.34 \\
\hline \multicolumn{4}{|l|}{ Sex: } \\
\hline Male & 2450 & 1 (referent) & NA \\
\hline Female & 1611 & 0.78 (0.67 to 0.91$)$ & 0.002 \\
\hline \multicolumn{4}{|c|}{ Primary renal diagnosis: } \\
\hline Non-diabetes & 3309 & 1 (referent) & NA \\
\hline Diabetes & 617 & 2.03 (1.68 to 2.46$)$ & $<0.001$ \\
\hline Missing & 135 & 0.63 (0.38 to 1.03$)$ & 0.07 \\
\hline
\end{tabular}

$\mathrm{NA}=$ not applicable.

NHS renal service commissioners, through an annual capitation fee paid by the renal centres submitting data. The Organ Donation and Transplant Directorate is a national body that administers organ donation and transplantation across the UK and records relevant information on patients on the waiting list as well as details of transplantation.

\section{Statistical analyses}

We developed a logistic regression model to identify the influence of patient specific variables including age, sex, ethnicity, and primary renal diagnosis on the probability of access to the transplant list and receipt of a transplant once on the waiting list. After adjusting for patient specific variables, we determined the percentage of patients activated on the transplant list as well as the percentage of patients on the waiting list who achieved a transplant in each centre. We assessed the overall effect of centre associated with each analysis by including renal centre as a random effect in the risk adjusted logistic regression model. We determined the extent of variation between centres by using a log likelihood ratio test that provides the change in the value of $-2 \log \mathrm{L}$ on inclusion of the random centre effect. ${ }^{16}$ We used SASv9.1 for analyses and considered a $5 \%$ level to be significant.

To analyse access to the transplant list, we identified the proportion of incident patients with end stage renal disease in each unit who were subsequently activated on the waiting list within two years of starting renal replacement treatment. We considered time to activation on the waiting list to be the interval between the start of renal replacement treatment and the date of activation on the waiting list. We estimated the median time to activation from the survivor function for patients at each renal unit, with the event as the date of activation and censoring at death or on 31
December 2007, whichever was earlier. We assessed the overall effect of centre associated with time to activation by including renal centre as a variable in a risk adjusted Cox regression model. To analyse the differences between centres in achieving a renal transplant, we estimated the percentage of patients activated on the waiting list who received a renal transplant within two years of being activated (conversion efficiency). We analysed separately the conversion efficiency for receiving a transplant from a donor after brain stem death or a donor after cardiac death/living kidney donor.

We represented the results for each outcome of interest by using funnel plots, which provide a visual comparison of each centre's performance compared with its peers. Where relevant, we adjusted the funnel plots for patient specific variables influencing that outcome. The solid black straight line in each funnel plot shows the overall average together with the $95 \%$ and $99.8 \%$ confidence intervals, which correspond to two and three standard deviations. Each point on the plot represents one renal centre. With 65 centres included, for each outcome of interest, two or three centres would be predicted to fall between the $95 \%$ and $99.8 \%$ confidence intervals (one above and one below) and no centre should fall outside the $99.8 \%$ confidence interval. We excluded centres with fewer than 10 patients starting dialysis $(\mathrm{n}=2)$ or fewer than 10 patients activated on the waiting list $(\mathrm{n}=6)$ during the study period from representation in the "percentage activated on the waiting list," "median time to listing," and "percentage transplanted" funnel plots.

\section{RESULTS}

Logistic regression model analysis of patient specific variables influencing access to the waiting list (table 1) showed a negative association with increasing age of recipient. The odds ratios were 0.75 (95\% confidence interval 0.61 to 0.93 ), 0.50 (0.41 to 0.61 ), 0.25 ( 0.20 to 0.30 ), and 0.11 (0.09 to 0.13 ) for patients in the age

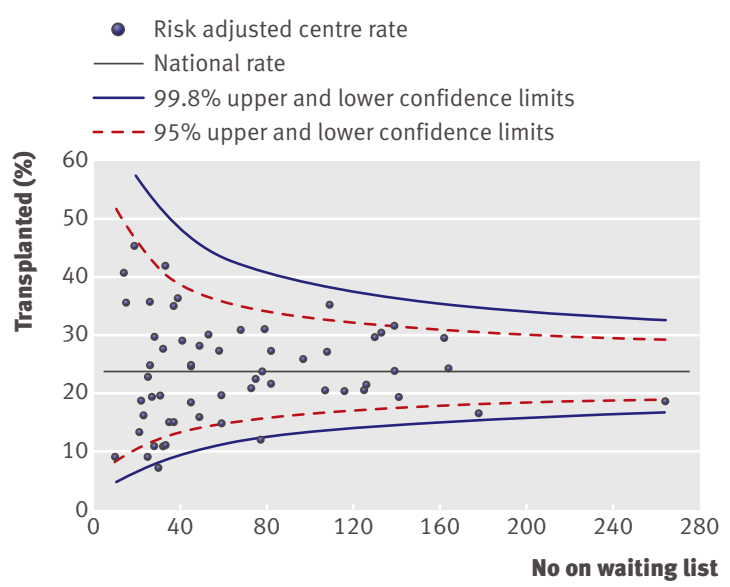

Fig 2 Percentage of patients receiving a transplant from a donor after brain stem death within two years of being registered for transplantation. Risk adjusted for effect of recipient's age, sex, ethnicity, and primary renal diagnosis 
Table 3 | Logistic regression modelling for probability of receiving transplant from donor after cardiac death or living kidney donor within two years of registration on waiting list

\begin{tabular}{|c|c|c|c|}
\hline Baseline factor & No & Odds ratio $(95 \% \mathrm{Cl})$ & $P$ value \\
\hline \multicolumn{4}{|l|}{ Age (years): } \\
\hline $18-29$ & 600 & 1 (referent) & NA \\
\hline $30-39$ & 853 & 0.58 (0.46 to 0.72$)$ & $<0.001$ \\
\hline $40-49$ & 1120 & $0.48(0.39$ to 0.60$)$ & $<0.001$ \\
\hline $50-59$ & 1062 & $0.33(0.26$ to 0.41$)$ & $<0.001$ \\
\hline $60-64$ & 426 & $0.28(0.21$ to 0.38$)$ & $<0.001$ \\
\hline \multicolumn{4}{|l|}{ Ethnicity: } \\
\hline White & 2645 & 1 (referent) & NA \\
\hline Non-white & 675 & $0.57(0.46$ to 0.71$)$ & $<0.001$ \\
\hline Missing & 741 & 0.88 (0.72 to 1.06$)$ & 0.18 \\
\hline \multicolumn{4}{|l|}{ Sex: } \\
\hline Male & 2450 & 1 (referent) & NA \\
\hline Female & 1611 & 0.99 (0.85 to 1.14$)$ & 0.85 \\
\hline \multicolumn{4}{|c|}{ Primary renal diagnosis: } \\
\hline Non-diabetes & 3309 & 1 (referent) & NA \\
\hline Diabetes & 617 & $0.48(0.38$ to 0.61$)$ & $<0.001$ \\
\hline Missing & 135 & 1.79 (1.24 to 2.59$)$ & 0.002 \\
\hline
\end{tabular}

$\mathrm{NA}=$ not applicable.

bands 30-39, 40-49, 50-59, and 60-64 years, compared with the age band of 18-29 years. We also found a negative association in patients who were non-white (odds ratio $0.85,0.75$ to 0.97$)$ or had missing ethnicity $(0.74$, 0.66 to 0.84 ) compared with white ethnicity. A primary renal diagnosis of diabetes (odds ratio 0.40, 0.36 to $0.45)$ or "missing" ( $0.55,0.43$ to 0.69$)$ was associated with a reduced likelihood of being put on the waiting list compared with patients with non-diabetic kidney disease. Sex had no influence on the probability of being activated on the waiting list.

Analysis of factors influencing the likelihood of receiving a transplant from a donor after brain stem death (table 2) showed that increasing age (odds ratios 0.69 (0.54 to 0.87 ) and 0.44 (0.31 to 0.61 ) for patients in age bands 50-59 and 60-64 years compared with age band $18-29$ years), non-white ethnicity $(0.47,0.37$ to 0.59 compared with white ethnicity), and female sex $(0.78,0.67$ to 0.91$)$ had negative associations. A primary renal diagnosis of diabetes (odds ratio 2.03, 1.68 to 2.46 ) had a positive association compared with a non-diabetes diagnosis for receipt of a transplant from a donor after brain stem death.

Similar analysis of factors influencing receipt of a transplant from a donor after cardiac death or a living kidney donor (table 3) showed a negative association with increasing age (odds ratios 0.58 ( 0.46 to 0.72 ), 0.48 ( 0.39 to 0.60$), 0.33$ (0.26 to 0.41 ), and 0.28 (0.21 to 0.38 ) for patients in age bands 30-39, 40-49, 50-59, and 60-64 years compared with age band 18-29 years). Non-white ethnicity (odds ratio $0.57,0.46$ to 0.71 ) and primary renal diagnosis of diabetes $(0.48,0.38$ to 0.61 ) were also negatively associated with receipt of a transplant from a donor after cardiac death or a living kidney donor, but we found no association with sex. These results suggest that non-white patients deemed suitable for renal transplantation are less likely to receive a transplant from either a donor after brain stem death or a donor after cardiac death/living kidney donor.

A patient starting dialysis in a non-transplanting renal centre was less likely to be registered for transplantation (odds ratio $0.85,0.77$ to 0.94 ) or receive a transplant from a donor after cardiac death or a living kidney donor $(0.69,0.59$ to 0.80$)$ compared with patients cared for in transplanting renal centres. Once registered for transplantation, patients in both transplanting and non-transplanting renal centres had an equal chance of receiving a transplant from a donor after brain stem death (odds ratio 1.01, 0.87 to 1.18 ).

We identified significant effects of centre for the probability of being activated on the waiting list (fig 1) (change in $-2 \log \mathrm{L}=89.9, \mathrm{df}=1, \mathrm{P}<0.001$ ) and the probabilities of receiving a renal transplant from a donor after brain stem death (fig 2) (change in $-2 \mathrm{Log} \mathrm{L}=15.1, \mathrm{df}=1, \mathrm{P}<0.001$ ) or a donor after cardiac death/living kidney donor (fig 3) (change in $-2 \log \mathrm{L}=46.1, \mathrm{df}=1, \mathrm{P}=0.001)$. These differences persisted even after adjustment for patient specific variables that were shown to affect the outcome event. As shown, several centres fall outside the $95 \%$ and $99 \%$ confidence intervals.

Figure 4 shows the unadjusted median time taken to activate patients on the transplant list for each centre. The funnel plot is based on the assumption of an exponential distribution for time to activation. Although this assumption is broadly consistent with the data, the model based estimate of the national median is greater than that observed. This leads to an unusually large number of units falling outside the lower $99.8 \%$ confidence limit for this national rate and perhaps too few occurring outside the upper limit. However, the plot highlights those centres that have significantly longer time to activation but small numbers of patients on the waiting list. A risk adjusted analysis of time to activation identified a significant effect of centre

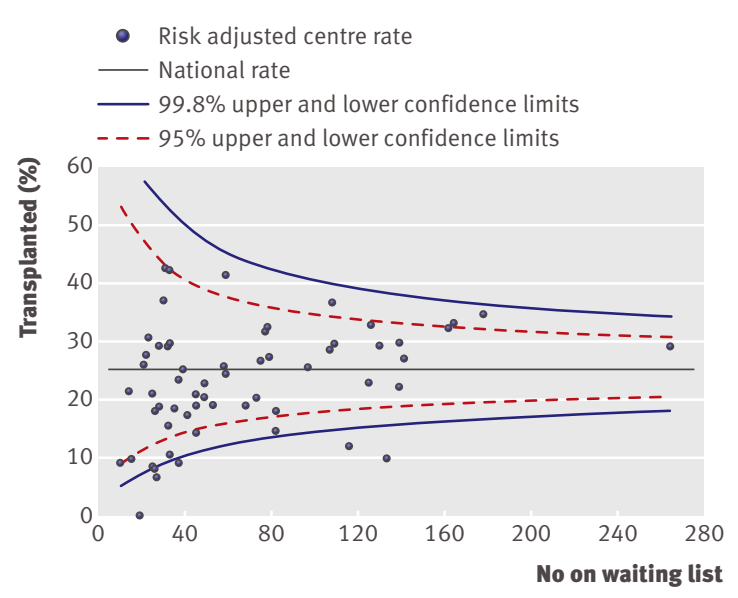

Fig 3 | Percentage of patients receiving a transplant from a donor after cardiac death or living kidney donor within two years of being registered for transplantation. Risk adjusted for effect of recipient's age, sex, ethnicity, and primary renal diagnosis 


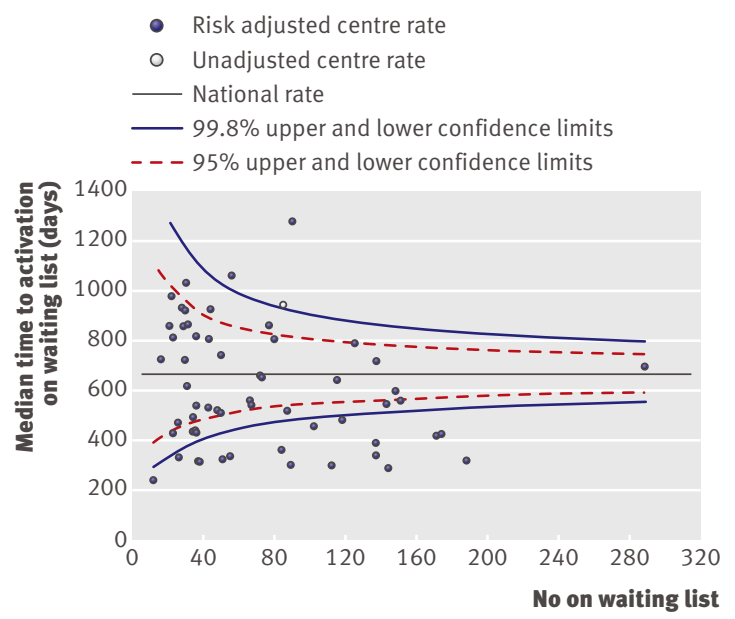

Fig 4 Median time to transplant listing after start of dialysis. Centre represented by unfilled symbol has final event time as plotting position, as median time for that centre could not be established with certainty

(change in $-2 \operatorname{LogL}=247.4, \mathrm{df}=64, \mathrm{P}<0.001$ ). Centres with the longest unadjusted waiting times also had the longest risk adjusted waiting times.

\section{DISCUSSION}

The main finding of our study is an effect of renal centre on patients' access to the national renal transplant waiting list - in the time taken to activate patients on the waiting list and in the receipt of transplantation once activated on the waiting list-and these differences cannot be explained by patient related factors (case mix). The patient related factors influencing access to the waiting list or receipt of transplantation identified in our study are consistent with those in previously published work. Inter-centre differences were more pronounced for access to transplants from donors after cardiac death and living kidney donors and the time taken to activate patients on the transplant list, outcomes that are often predominantly influenced by individual centres' practices and policies.

\section{Strengths and limitations of study}

Although previously published literature clearly highlighted patient specific variables that influence access to renal transplantation, other national renal and transplant registries have not been able to describe differences at the level of the centre. The strength of this study is in the absence of introduction of potential bias by recruiting all incident patients starting renal replacement treatment. In addition, no patients were lost to follow-up. Lack of comprehensive comorbidity data on all patients is a potential weakness of our study. In the absence of comprehensive patient level data permitting definitive adjustment for case mix, our results need to be interpreted with caution, as patient related factors other than those analysed as part of the study may be important in influencing access to renal transplantation. Some centres may take on "sicker" patients with higher comorbidity, explaining some of the observed inter-centre variability. However, a recent analysis from the UK Renal Registry reporting on one year mortality in incident renal replacement treatment patients from the same study period identified much less variability in patients' survival, ${ }^{17}$ and centres performing less well on the survival analysis were not the same as the centres outside the lower $99.8 \%$ confidence limit in the analysis of access to transplant. Even though not conclusively established as part of our study, this suggests that factors other than comorbidity may exert a significant influence on the probability of patients starting renal replacement treatment accessing the transplant waiting list or receiving a renal transplant in the UK. Many factors within and outside of the centre's control will influence the rate of transplantation from donors after brain stem death. For example, the change to the national kidney allocation scheme in April 2006, after which time both kidneys rather than just one from each donor were allocated on a national basis, as well as the increased priority given to time on the waiting list to determine organ allocation, will affect centres' transplant rates. These changes were introduced to improve the chances of ethnic minorities and other long waiting patients receiving a kidney from a donor after brain stem death, among other reasons. Our analysis adjusts for ethnicity over the entire study period, but adjustment for the effect of all of the changes in the allocation scheme on centre specific transplant rates before and after April 2006 would not be possible.

\section{Comparison with other studies}

Two previous studies have attempted to explore the potential for variations in access to transplantation in the UK. The study by Oniscu et al was similar to our study in that it included incident patients starting renal replacement treatment and assessed patient level and centre level variables influencing access to waiting list activation as well as receipt of a transplant. ${ }^{13}$ However, this study was restricted to patients in Scotland and was from an era in which patients declared brain stem dead were the predominant source of donor organs. Dudley et al reported on point prevalence analysis and included patients from 41 renal units in England and Wales. ${ }^{14}$ This study included a large number of patients and also assessed the influence of factors such as social deprivation on access to the transplant waiting list. Point prevalence results from that study could be influenced by short term fluctuations in numbers of patients and selective enrichment of the pool of prevalent patients with patients deemed unsuitable for transplantation, as well as by individual centres' transplant rates. For these reasons, point prevalence data should be interpreted with caution. Neither of the above studies included analysis of time taken to place patients on the waiting list.

\section{Policy implications}

The finding that certain patient related variables such as increasing age have a negative association with access to transplantation is understandable, as the 


\section{WHAT IS ALREADY KNOWN ON THIS TOPIC}

Patient specific variables such as age and comorbidity affect access to renal transplantation for patients with end stage renal disease

In healthcare systems elsewhere in the world, patient independent variables such as insurance status are also known to affect access to renal transplantation

\section{WHAT THIS STUDY ADDS}

Variations exist in access to the renal transplant waiting list, time to inclusion on the waiting list, and receipt of a renal transplant in the UK, which cannot be explained by variations in case mix

Significant inter-centre variability was seen in outcomes predominantly under the influence of centre specific practices, such as time to waiting list inclusion and transplantation from donors after cardiac death/living kidney donors

risk-benefit ratio of receiving a renal transplant alters with age. However, the effect on access to transplantation of factors such as sex and ethnicity is more difficult to understand. The importance given to HLA matching in the national allocation protocol at the time of this study would have favoured a predominantly white donor pool being matched with white recipients, which may explain the effect of ethnicity on this outcome. We have not analysed the interplay between factors such as social deprivation and ethnicity and whether the observed differences based on ethnicity are likely to persist after adjustment for social deprivation and varying comorbidity burden in different ethnic groups. One possible explanation for the observed disparity between the sexes in receipt of a transplant from a donor after brain stem death could be pregnancy related HLA sensitisation in women, which in turn will limit offers of organs. The higher proportion of patients with diabetes receiving a transplant corresponds to an increase in the number of simultaneous kidney-pancreas transplants done during the study period, as the allocation algorithm prioritised dual organ recipients.

More importantly, our study highlights the presence of a significant effect of centre in access to transplantation, even after correction for the relevant patient related variables. However, concluding that centres with a lower proportion of patients on the waiting list are in some way performing less well would be simplistic. Such centres could be choosing patients more carefully to ensure that the scarce resource of donated organs is appropriately targeted to patients who are likely to benefit the most. Centres with the highest proportion of patients on the waiting list may be including patients who have a higher risk of peri-operative morbidity or mortality and hence may have inferior posttransplant outcomes. This would represent suboptimal use of the scarce resource of donated organs, but the lack of an effect of centre on post-transplant survival of patients and grafts argues against this explanation (www.uktransplant.org.uk/ukt/statistics/centre-speci fic_reports/kidney_centre-specific_reports.jsp). For these reasons, in the absence of objective guidance dictating inclusion and exclusion thresholds to determine access to transplantation for patients starting renal replacement treatment, prescribing the minimum percentage of patients who should be activated on the renal transplant waiting list in every centre would be difficult. However, significant inter-centre differences should not exist in the time taken to activate suitable patients for transplantation or in receipt of a transplant once on the waiting list, especially for transplantation from donors after cardiac death or living kidney donors, as centres' practice patterns are predominantly responsible for these outcomes. Best practice identified in the better performing centres needs to be disseminated to all centres to enable equity of access to transplantation across the UK.

\section{Conclusions}

Further work needs to be done to determine whether the observed differences in centres' performance are due to variations in availability of resources or because certain centres have more organised and efficient pathways for patients. A qualitative analysis of centres' practices that act as enablers or barriers for efficient progression of a patient with a new diagnosis of severe renal impairment through to receiving a renal transplant will result in more uniform access to renal transplantation irrespective of where patients start their renal replacement treatment. The development of a survival probability tool or more sophisticated tools to estimate gain of quality adjusted life years would enable the development and implementation of more objective guidance for patients on the potential benefits of renal transplantation.

Contributors: RR had the idea for the study, developed the methods and analysis, and wrote the manuscript. UU, DA, CRVT, and CRKD developed the methods, were involved in discussions of the analysis, and contributed to writing the manuscript. UU and DA obtained the data from the UK Renal Registry. DC, RJ, JO'N obtained the data from the UK Transplant Registry, were involved in developing the methods and discussions of the analysis, did the statistical analysis, and contributed to writing the manuscript. RR is the guarantor

Funding: None

Competing interests: All authors have completed the Unified Competing Interest form at www.icmje.org/coi_disclosure.pdf (available on request from the corresponding author) and declare that (1) None of the authors have received support from any source for the submitted work; (2) None of the authors have any relationship with any company or funding source that might have an interest in the submitted work in the previous 3 years; (3) their spouses, partners, or children do not have any financial relationships that may be relevant to the submitted work; and (4) All authors have no non-financial interests that may be relevant to the submitted work.

Ethical approval: Not needed. The paper contains no identifiable patient information. Both the UK Renal Registry and UK Transplant Registry are regulated under the NHS Act 2006 section 251 and have been granted permission by the Secretary of State to collect identifiable patient data without individual patient consent.

Data sharing: A technical appendix, statistical code, and full dataset are available from JO’N (John.O’Neill@nhsbt.nhs.uk).

1 Bloembergen WE, Mauger EA, Wolfe RA, Port FK. Association of gender and access to cadaveric renal transplantation. Am J Kidney Dis 1997;30:733-8.

2 Garg PP, Furth SL, Fivush BA, Powe NR. Impact of gender on access to the renal transplant waiting list for pediatric and adult patients. J $\mathrm{Am}$ Soc Nephrol 2000;11:958-64.

3 Cass A, Cunningham J, Snelling P, Wang Z, Hoy W. Renal transplantation for indigenous Australians: identifying the barriers to equitable access. Ethn Health 2003;8:111-9. 
4 Bayat S, Frimat L, Thilly N, Loos C, Briancon S, Kessler M. Medical and non-medical determinants of access to renal transplant waiting list in a French community-based network of care. Nephrol Dial Transplant 2006;21:2900-7.

5 Alexander GC, Seghal AR. Barriers to cadaveric renal transplantation among blacks, women, and the poor. JAMA 1998;280:1148-52.

6 Abbott KC, Glanton CW, Agodoa CY. Body mass index and enrollment on the renal transplant waiting list in the United States. J Nephrol 2003;16:40-8.

7 Gaylin DS, Held PJ, Port FK, Hunsicker LG, Wolfe RA, Kahan BD, et al. The impact of comorbid and sociodemographic factors on access to renal transplantation. JAMA 1993;269:603-8.

8 Axelrod DA, Guidinger MK, Finlayson S, Schaubel DE, Goodman DC, Chobanian M, et al. Rates of solid-organ wait-listing, transplantation, and survival among residents of rural and urban areas. JAMA 2008;299:202-7.

9 Sequist TD, Narva AS, Stiles SK, Karp SK, Cass A, Ayanian JZ. Access to renal transplantation among American Indians and Hispanics. Am J Kidney Dis 2004:44:344-52.

10 Wolfe RA, Ashby VB, Milford EL, Bloembergen WE, Agodoa LY, Held PJ, et al. Differences in access to cadaveric renal transplantation in the United States. Am J Kidney Dis 2000;36:1025-33.
11 Thamer M, Henderson SC, Ray NF, Rinehart CS, Greer JW, Danovitch GM. Unequal access to cadaveric kidney transplantation in California based on insurance status. Health Serv Res 1999;34:879-900.

12 Alexander GC, Seghal AR. Variation in access to kidney transplantation across dialysis facilities: using process of care measures for quality improvement. Am J Kidney Dis 2002;40:824-31.

13 Oniscu GC, Schalkwijk AA, Johnson RJ, Brown H, Forsythe JL. Equity of access to renal transplant waiting list and renal transplantation in Scotland: cohort study. BMJ 2003;327:1261.

14 Dudley CR, Johnson RJ, Thomas HL, Ravanan R, Ansell D. Factors that influence access to the national renal transplant waiting list. Transplantation 2009;88:96-102.

15 Meier-Kriesche HU, Kaplan B. Waiting time on dialysis as the strongest modifiable risk factor for renal transplant outcomes: a paired donor kidney analysis. Transplantation 2002;74:1377-81.

16 Collett D. Modelling binary data. 2nd ed. CRC Press, 2002.

17 Ansell D, Roderick P, Hodsman A, Ford D, Steenkamp R, Tomson C. UK Renal Registry 11th annual report. UK Renal Registry, 2008.

Accepted: 30 April 2010 